\title{
Spintronic Materials and Devices: Advances and Applications
}

\author{
Ashutosh Tiwari
}

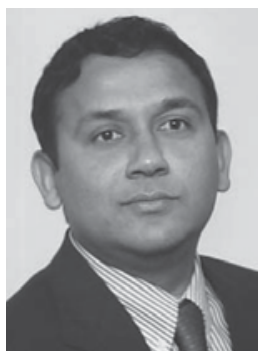

\section{Advances in} present-day microelectronics and magnetic data storage devices depend critically on the technological ability to produce device struc-

tures of ever-decreasing dimensions. With the typical device dimensions approaching nanometer scale, the materials design parameters are being pushed to their optimum values. Continued device miniaturization is becoming increasingly challenging. There is now a general consensus that the current rate of miniaturization cannot be sustained and new approaches of device fabrication need to be developed. Spintronics is one such approach that is based on the synergetic use of charge and spin dynamics of electrons in certain multifunctional materials.

There are various novel device concepts which have been proposed based on this technology, and a few (e.g., read heads for magnetic recorders and nonvolatile memory components) have already found practical applications. The critical step in the functioning of a spintronics device is the injection and detection of spin-polarized carriers at the ferromagnet-semiconductor interface. Despite considerable efforts, efficient injection of spins into nonmagnetic semiconductors still continues to be a major obstacle in this field. Ferromagnetic metals such as iron and nickel were used in earlier studies for injecting spin-polarized carriers into a semiconducting transport medium. However, in most of the studies the degree of spin polarization of injected carriers was less than a disappointing $1 \%$. In order to overcome this, three different approaches have been proposed for the efficient injection of spin-polarized carriers into semiconductors: tunneling spin-polarized carriers from ferromagnetic metals into the semiconductor through an insulator, tunneling non-polarized carriers through ferromagnetic insulator, which acts as a spin filter, and using dilute magnetic semiconductors (DMS) which are ferromagnetic and whose conductivity can be tuned to match the nonmagnetic semiconducting transport medium.

All of the techniques that are listed above have been widely tested by several research groups for different applications. Close to $30 \%$ spin-polarized injection using the first approach has been reported in literature. Compared to this, reasonably higher spin-polarized carrier injection can be achieved using the latter two approaches. But for efficient spintronics devices, it is desirable to have nearly $100 \%$ spin-polarized carrier injection. So, it is widely believed that any further progress in spintronics will heavily rely on the rapid developments in fabricating new and novel classes of DMS and diluted magnetic insulators/dielectrics (DMD). A stringent requirement for these materials is that they should be ferromagnetic at room temperature (i.e., the ferromagnetic transition temperature $T_{C}$ should be more than $300 \mathrm{~K}$ for the practical realization of devices).

The article by John Paul Wallace shows that the response of iron and steel to a time-dependent magnetic field at room temperature can drive the formations of Bose-Einstein-like condensations (BELC) at the applied frequencies and generate multiple propagating modes. The formalism adopted is to apply the Gross-Pitaevskii equation to Bose-Einstein condensation (which is a non-linear variation of the Schrodinger equation).

The article by M. Snure et al. reports the progress in $\mathrm{ZnO}$-based diluted magnetic semiconductors. $\mathrm{ZnO}$-based DMS have attracted a great deal of research attention and controversy over the past decade. The initial attention was sparked by the prediction of above oom-temperature ferromagnetism in manganese-doped $\mathrm{ZnO}$ by Dietl. This was followed by a surge of reports of ferromagnetism in thin film transition metal (TM)-doped ZnO. However, reported values of magnetic moments and Curie temperatures were inconsistent, which led to controversy over the origin of the observed ferromagnetism. In this paper the authors reviewed a number of TM-doped $\mathrm{ZnO}$ based DMS in order to clarify which materials are likely to be intrinsically ferromagnetic.

The article by J. Narayan et al. reports the investigations on the Ni-MgO DMD system with a nickel concentration of 0.5 at. $\%$. They showed that in as-grown crystals, nickel ions occupy substitutional magnesium sites. Under these conditions, the $\mathrm{Ni}-\mathrm{MgO}$ system behaves as a perfect paramagnet. By using a controlled annealing treatment in a reducing atmosphere, they were able to induce clustering and form pure nickel precipitates in the nanometer size range. The spontaneous magnetization data for the samples, where nickel was precipitated randomly in an MgO host, fits well to Bloch's $\mathrm{T}^{3 / 2}$-law and has been explained within the framework of spin wave theory predictions.

AshutoshTiwari is with the Department of Materials Science and Engineering at the University of Utah, SLC, Utah 84112, USA and is the advisor to JOM from the Superconducting and Magnetic Materials Committee of the Electronic, Magnetic \& Photonic Materials Division of TMS. 